\title{
Modeling evacuation dynamics on stairs by an extended optimal steps model
}

\author{
Yiping Zeng ${ }^{1,3}$, Weiguo Song ${ }^{1, *}$, Feizhou $\mathrm{Huo}^{2}$, Giuseppe Vizzari ${ }^{3}$ \\ ${ }^{1}$ Sate Key Laboratory of Fire Science, University of Science and Technology of China, Hefei 230027, People's Republic of China \\ ${ }^{2}$ Wuhan University of Technology, Number 205, Luoshi Road, Hongshan District, Wuhan, 430070, PR China \\ ${ }^{3}$ Complex Systems and Artificial Intelligence research center, University of Milano-Bicocca Viale Sarca 336-Ed. U14, 20126 \\ Milano(ITALY)
}

\begin{abstract}
:
With more and more high-rise building springing into the cities, the movement of pedestrians on stairs is of great importance for the evacuation of these facilities; the topic of human performance on stairs has attracted a lot of researcher to study. However, there is still a lack of analysis of specific situations, such as mid-landing and merging behaviors. In this paper we employ a modified version of the Optimal Steps Model, modified by taking block-based floor field into consideration under open boundary conditions, to reproduce movement at mid-landing and study performance of occupants in stairwell. Movements on this kind of stairs are simulated and studied with the help of extended model: achieved results are in accord with previous research in respect of specific flow and fundamental diagram. Lane formations on stairs come into being before mid-landing area through simulations. Furthermore most people reach at the mid-landing by the outside stairs at higher density due to more space and less force from other agents, which is induced that outside of the stairs is good for evacuation. At last the model is implemented to study the effect of stairs geometry on merging behaviors, and it is found when corridor is connected to the landing opposite to the incoming stairs, this structure is biased in favor of occupants from stairs. The work in this paper is intended to better understand movement during stair evacuations and develop a technical foundation for codes and standards requirements as well as egress modeling techniques.
\end{abstract}

Keywords: stairs movement; merging behaviors; lane formation;

\footnotetext{
*Weiguo song(囚)

State Key Laboratory of Fire Science, University of Science and Technology of China, Hefei 230026, P.R China

E-mail address:wgsong@ustc.edu.cn
} 


\section{Introduction}

With the development of society, more and more high-rise buildings are shown in the cities in the past decades. Although the high-rise building help occupants provide more area to live in and save a lot of landing, the attentions are focused on the problem how to evacuate out of the high-rise building in a minimum time. This problem results from the following factors: 1) it is a common sense that it is not safe to use elevators in emergency [1] and occupants are educated that elevators are not allowed to use; 2) it is noted that some laws [2,3] forbid occupants to use elevators under the condition of emergency such as fire, earthquake and so on; 3) the stairwell is limited to the space in high-rise building due to the cost of buildings; 4) congestions are more likely to occur on the staircase of the building and densities at merging area increase easily due to flows from upper stairs and floors [4]. For all these reasons stair evacuation plays a crucial role in building evacuation and stairs have attracted a lot of researchers to study their influence on pedestrian behavior by means of experiments and simulations.

On the side of acquisition of data about the phenomenon, observations [5, 6], controlled experiments $[7,8]$ and fire drill experiments $[9,10]$ were carried out to analyze the performance of occupants on stairs in terms of fatigue, stair width, merging, counter-flow and so on. On the side of the synthetic reproduction of pedestrian behavior, to support planning, there are several models to reproduce the movement of human agents in computers. BuildingExodus is used to investigate the characteristics in stairwell [11], and Galea et al reproduced the merging behaviors when occupants were confronted with each other at the mid-landing [12] and he found that when incoming flow was adjacent to the floor, occupants had advantage to evacuate. Finite element models were also used to study movement on stairs due to the detailed description of occupants in simulation [13, 14]. Qu et al. [15] tested the validity of the model and then he used the extended social force model to analyze the effect of stair geometry and optimal velocities. Based on the social force model Anylogic, Hou et al. [16] investigated the influence of stair slope and velocity on evacuation, and they found that in the large scale evacuation problem the total evacuation time could not be reduced limitlessly as the evacuation velocity speeded up. Huo et al. [17] analyzed the merging phenomenon at the floor-stair interface with the help of an extended CA model. Ding et al. [18] took the preference of occupants into consideration and reproduced the movement in experiments by applying preferences into CA model. Michael et al. [19] developed an optimal steps model in which occupants could be modelled in continuous space; following extensions were aimed at adjusting dynamic stride length according to utility and personal space [20], and to represent movements in stairs [21]. In particular, the last work correctly reproduced observed behaviors and the fundamental diagram.

To date, OSM [19] takes advantage of characteristics of social force and CA models, and it is widely validated and accepted in the case of corridors, bottlenecks, stairs and so on. However, there is still a lack of knowledge about the research on stairs with midlanding and merging behaviors. The present work considers pedestrian vertical 
movement and merging flow at the mid-landing: the OSM model is modified by taking block-based floor field into consideration under open boundary conditions, with the aim to reproduce movement at mid-landing and study performance of occupants in stairwell. To describe the work in this paper, the original OSM model and the proposed modifications are briefly introduced in terms of basic movements, evacuee setup floor field and update rules in section 2; in section 3, the model is tested to validate fundamental diagram and specific flow, then lane-formation and merging phenomena are investigated. Conclusions are given at last section.

\section{Stairs model}

The extended stairs model is mainly developed based on Optimal Steps Model, and each agent is considered as a virtual pedestrian with distinct physical and psychological characteristics in the model. In Optimal Steps Model [19], a local circle is discretized into 20 parts in this paper by considering horizontal movements on stairs (as shown in Fig. 1). This allows for movement in arbitrary directions, only limited by the chosen optimization algorithm and numerical resolution. Furthermore, occupants move in a continuous space. More details about the model are shown in the following sections.

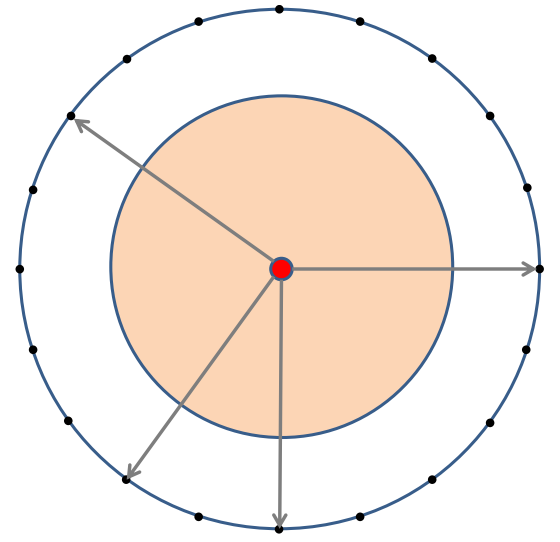

Fig. 1 Discretization of the circle with 20 points and four arrows showing the potential moving directions after optimization algorithm and numerical resolution

\subsection{Basic movement rules of pedestrians}

In contrast to cellular automata, the Optimal Steps model does not represent pedestrians by cells, nor do they have to move from cell to cell, which could hold one agent at most[22]. Pedestrians are represented by their position and extension in space [19] and they move by quantifying the effect of the agent's presence in the nearby environment. The force perceived by a pedestrian $l$ in coordinates $(x, y)$ is described by the following formula: 


$$
P_{l}(x, y)=P_{t}(x, y)+\sum_{i=1, i \neq l}^{n} P_{p, i}(x, y)+\sum_{j=1}^{m} P_{o, j}(x, y)
$$

$P_{p, i}(x, y)$ is the repulsive potential of pedestrian $i$ on a pedestrian in position $(x, y)$, $P_{o, j}(x, y)$ is indicated as the repulsive potential induced by obstacle $j$; a target potential value $P_{t}(x, y)$ can be interpreted as the occupants' orientation to exit or more generally the ability to move forward the lower floors. An example situation clarifying the different involved factors in defining the overall forces driving the pedestrian is shown in Fig. 2.

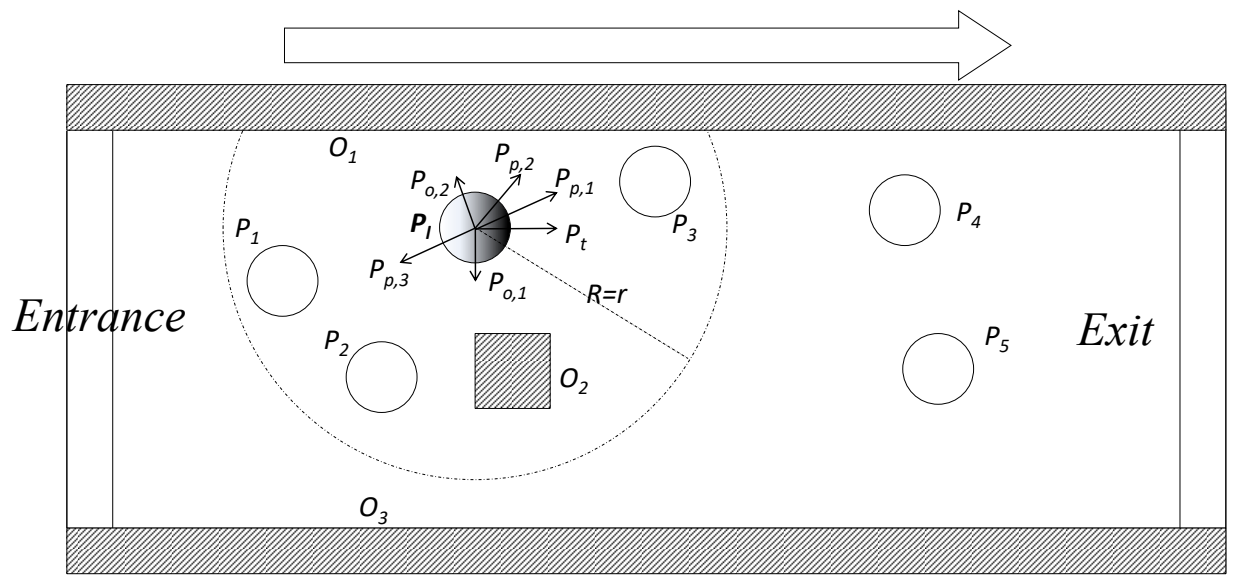

Fig. 2 A simple example for occupant affected by the environment

\subsection{Set up of evacuee agent}

In many traditional discrete models [23], to reproduce the realistic movement in simulations, a pedestrian occupies a square space whose widths are about $40 \mathrm{~cm}$ or 50 $\mathrm{cm}$ sided square cells. Furthermore, in many models, the torso of pedestrian is set up as $40 \mathrm{~cm}$. In addition, the average shoulder width for Chinese males and females during 18 - 60 years old are $41.5 \mathrm{~cm}$ and $38.7 \mathrm{~cm}$ referring to China National Standard Human Dimensions of Chinese Adults (GB10000-88) [24]. The average shoulder width for all Chinese is calculated as $40.1 \mathrm{~cm}$. The factors mentioned-above are taken into consideration so that the torso of pedestrian in this paper is set as $40 \mathrm{~cm}$. All step lengths are set as a constant value $40 \mathrm{~cm}$ in this model. This depends on the following reasons:1) in the analysis of Fang [25], the step length ranges from $0.2 \mathrm{~m}$ to $1.0 \mathrm{~m}$; 2) smaller step length can help obtain higher density; 3 ) the length of tread in most stairs are smaller than $0.4 \mathrm{~m}[15,26,27]$. Due the fact movement in stairs is limited to the width of tread, the step length on stairs counts on the following mechanism [21] in Fig. 3. Occupants start to move with the help of the equation 1, but step length is changed according to the width of tread and its own step length. 


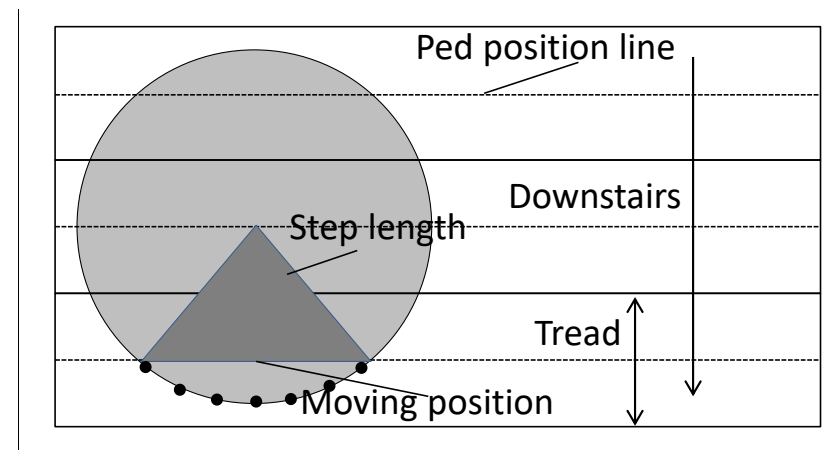

Fig. 3 Occupant movements on stairs: occupant moves forward according to the directions of the black points.

Previous researchers $[28,29]$ thought that the movement in the stairwell should be divided into two parts: the on-landing movement and on-stair movement. Movement at the mid-landing is the normal behavior on horizontal ground. However the movement on stairs is based on different mechanism, and the performance is potentially affected by the riser and tread depth, as well as the slope value of the stairs [4, 30-32]. Experiments and fire drills research indicated that speeds of agents on stairs had a relationship with the slope when individual differences were not considered and could be expressed as the following formula [26, 33]:

$$
v_{s}=v_{h} \cos \theta
$$

$v_{s}$ represents the velocity on stairs while $v_{h}$ denotes the horizontal velocity on floor or mid-landing; as for the $\theta$, it is the angle of the stairs, shown in Fig. 4

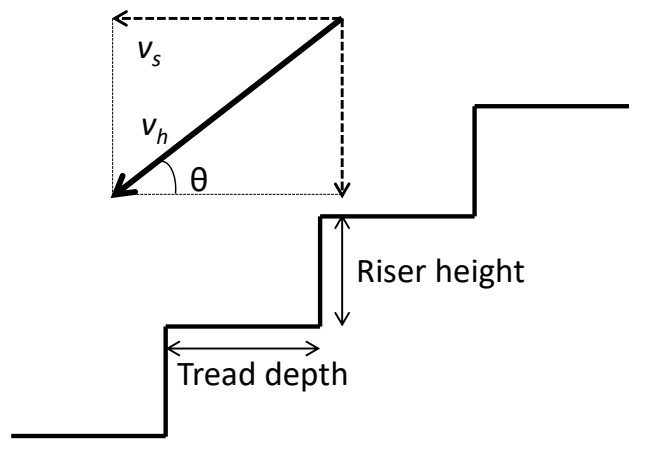

Fig. 4 Illustration of the rules of walking on the stairs

\subsection{Floor field}

Whereas the previous section describes the basic OSM model, we introduce an original modification, aimed at achieving a smooth trajectories in lands. An attractive force to the target is realized with a potential that represents the propagation of a wave front and is implemented as a floor field [34]. In this model, Bilinear interpolation is used into model to obtain a target potential value $P_{t}(x, y)$ for an arbitrary point $(x, y) x^{*} y$ $\in R^{*} R$ in the plane. As for the floor field during the movement in stairwell, floor field 
should be divided into two parts: stair field (shown in Fig. 5(a)) and mid-landing corner field (shown in Fig. 5(b)). When occupants walk down on stairs, it is not necessary to turn around or change the directions (unless additional conditions are faced, e.g. obstacles, counter flows), and pedestrians just keep walking down at the desired velocity to get out of the stairs in a minimum time. So the static floor is calculated by fast marching method on a two-dimensional grid [35, 36], and agent is mainly dominated by static floor without any pedestrians or obstacles around the agent. For floor-stair interface [37], a pedestrian usually changes his/her velocity and direction to make a turn by a center point with a certain turning radius, in order to adapt the movement at the mid-landing and pass through the corner with comfortable ways (shown in Fig. 5(b)). Templer et al. [38] and Hyun-seung et al.[39] found that when occupants passed through a corner at the mid-landings, the trajectories of the movement were similar to a semi-circle. Thus the floor field at corner is defined by the following studies [37]. First of all, the corner is divided into $2 * n$ parts, then the value of potential line $l_{k}$ is calculated as the following formula:

$$
S_{l_{k}}=\frac{k}{2 n} \phi \max (\text { length, width }), 0 \leq k \leq 2 n
$$

Where the $k$ is the label of each part as shown in Fig. $6 ; \phi$ is the total angel value for the analyzed corner, when it comes to the L corner, the value $\phi$ is $\pi / 2$ while the value is $\pi$ for the $U$ shape corner. Length and width are shown in Fig. 6.

In this framework, finally, the floor field of grid $(i, j)$ is calculated:

$$
S_{(i, j)}=\frac{\sum\left(S_{l_{k}} \cdot l_{k}^{(i, j)}\right)}{\sum l_{k}^{(i, j)}}
$$

The $l_{k}^{(i, j)}$ is the line length located at the cell $(i, j)$ by line $l_{k}$, as shown in Fig. 6 .

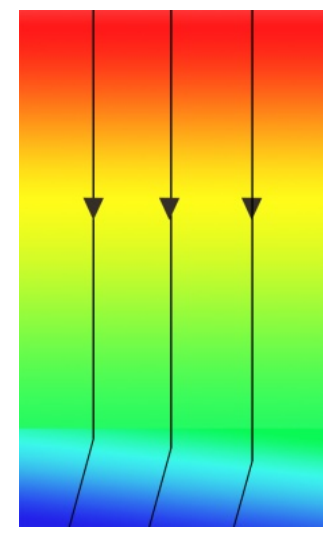

(a)

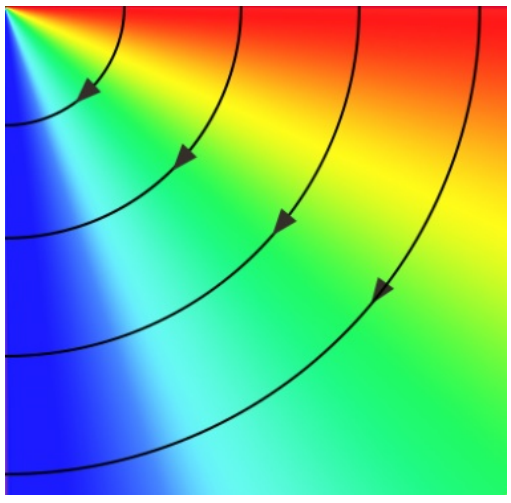

(b)

Fig. 5 Illustration of floor field in respect of stair(a) and mid-landing corner(b) 


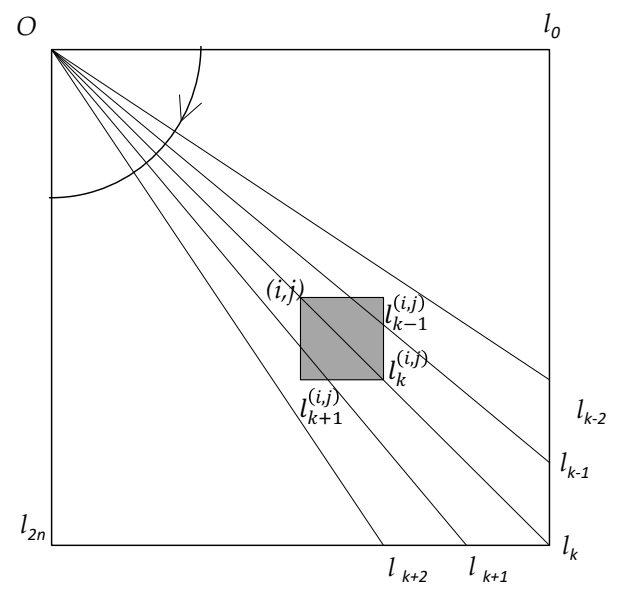

Fig. 6 Illustration of floor field calculation at the mid-landing corner

\subsection{Update rules}

To reproduce the realistic human movement, an event-driven update rule [40] is introduced in this model. In event-driven updates, movements of occupants do not depend on a unit time clock step according to some fixed or random order, and the occupants walk to next step in their natural order. The next step is related to unit time step length $\Delta t$ and individual time credit $\tau$. In the model, every pedestrian has an individual time credit $\tau$, movement step length $\lambda$ and desired speed v. Once the model is activated, the individual time credit $\tau$ is increased with a unit time step $\Delta t$ according to the step update. Each pedestrian is allowed to move to the next step only when the time credit is larger than the value of step length $\lambda$ divided by the desired speed $v$. To sum up, the occupants move at each time step according to the following mechanism shown in Fig. 7. In previous section, we set the step distance for $40 \mathrm{~cm}$, and this is the right value also for $\lambda$. We take velocities of $1 \mathrm{~m} / \mathrm{s}$ as an example (in the simulation velocity can actually be different), and $\Delta t$ is set for $0.4 \mathrm{~s}$.

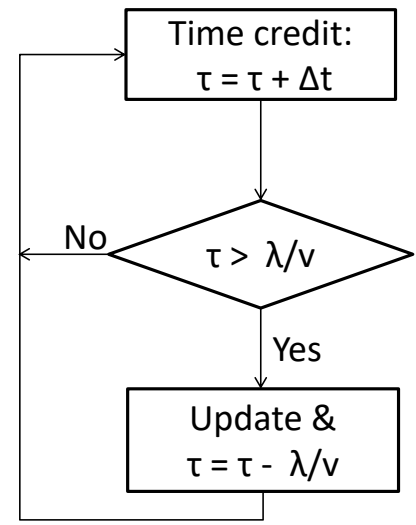

Fig. 7 Event-driven algorithm flow chart

Fig. 8 shows the agent interacting with the environment around the agent. When program starts, students are initialized at the start area and given a random location by 
the system; With increase of the time, each pedestrian is activated by comparing the accumulated individual time credit $\tau$ and unit time step $\Delta t$; Once activated, the agent could be considered as a virtual pedestrian and is simulated accordingly with distinct physical and psychological characteristics and objectives. Thus, pedestrian is affected with the impact of obstacles and other pedestrian around the agent. After recognizing the existing obstacles and pedestrians within some range, the values of floor field in 20 directions are obtained with the help of equation (1). Each pedestrian prefers the direction with less influence of obstacles and pedestrians, and at the same time this direction is closer to the lower floor. According to the movement direction, the agent walks with a step length on interface or takes a step on the stairs. If all directions are occupied by other occupants the occupant stands still and does not move at any directions.

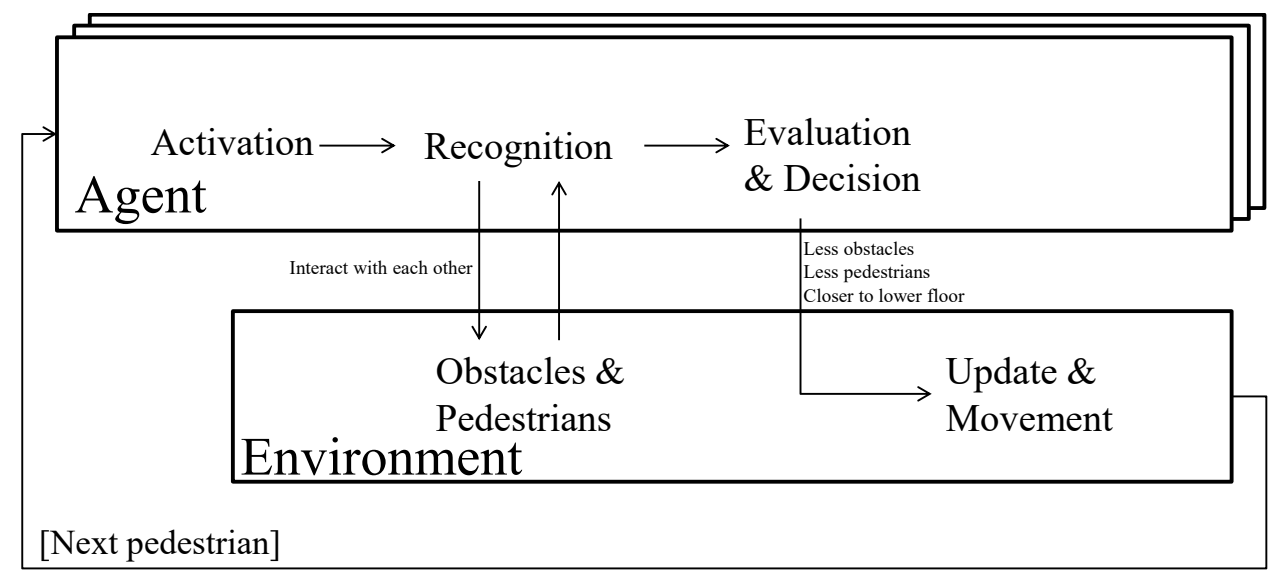

Fig. 8 Agents movement flow chart

\section{Results and analysis}

For the purpose of avoiding the influence of uncertainty, the model is simulated with different initial densities at the start area to evaluate the validation of model. And each scenario was simulated 10 times. The input parameter of the model contains the moving speed, the geometry of staircase and interface and so on. The results and analysis are shown and discussed in the following parts.

\subsection{Validation based on fundamental flow characteristics}

In order to investigate the performance of the model, one of the best ways is to reproduce the scenario and compare the data from simulations with previous results. In previous studies, many researchers [29, 41, 42] carried out controlled experiments and observed experiments to obtain the fundamental human behaviors when they walked down stairs. Thus in this paper, the scenario is simplified because more attention is paid to fundamental diagram and specific flow in the landing area and the simulation are carried out with the scenario as shown in Fig. 9. Pedestrians are initiated at the start 
area of stairs, and each agent is removed out of the system when occupants arrive at the end area. Simulation is reproduced with 3000 time steps ( $0.1 \mathrm{~s}$ for each time step) and the values from 100s to 250s (shown in Fig. 10) are taken into consideration because that during this period there is a steady state in respect of density and specific flow.

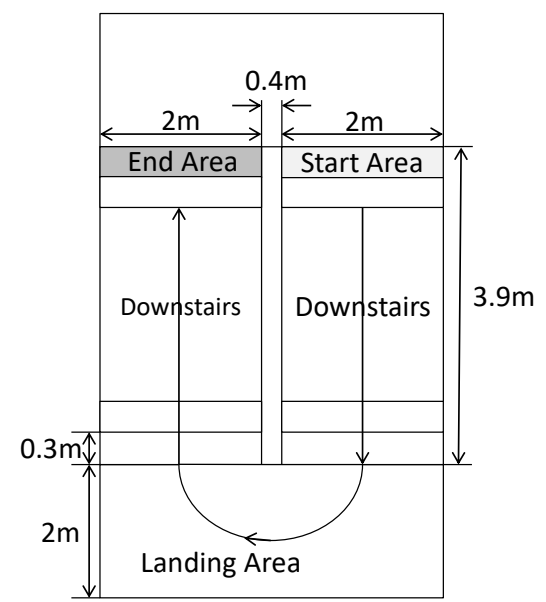

Fig. 9 Simulation Scenario

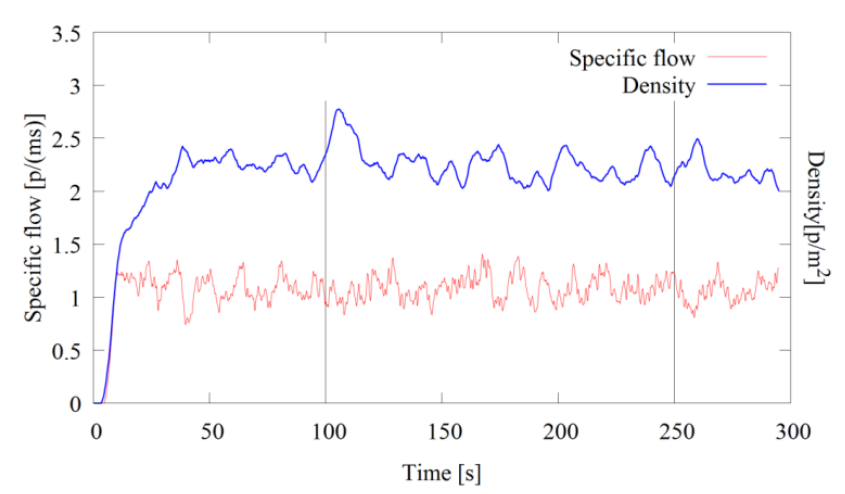

Fig. 10 Fundamental characteristic with time

The measurements of this model are obtained with the help of velocity and stairs slope in Burghardt et al. [29]. In [29], the biggest horizontal velocities range from $0.6 \mathrm{~m} / \mathrm{s}$ to $0.9 \mathrm{~m} / \mathrm{s}$ when occupants walk down stairs, then the desired horizontal speed of Nelson and Mowrer (NM) [43], Weidmann (WM) [44] are about $0.70 \mathrm{~m} / \mathrm{s}$ and 0.80 $\mathrm{m} / \mathrm{s}$, separately, as shown in Fig. 12(a). Thus the average value $0.75 \mathrm{~m} / \mathrm{s}$ is considered as an input parameter in model and the slope value 32.7 degrees is inserted into the model. After simulation and calculation at the mid-landing, the corresponding results are shown in Fig. 11. Label "model" (red stars) means that the value calculated by our model and label "calculated" represent the values obtained by timing density and horizontal speed, which is the way to obtain specific flow in [29]. It is easily noted that the simulation results are in accord with the data of Burghardt et al. [29] in terms of fundamental diagram and specific flow. According to the model simulation, the largest horizontal velocity is about $0.94 \mathrm{~m} / \mathrm{s}$ when density is about 0.1 and smallest speed is $0.4 \mathrm{~m} / \mathrm{s}$ at density $2.8 \mathrm{p} / \mathrm{m} 2$. Due to the fact there is a potential repulsive force among 
pedestrians, the agent tries to keep distance from people surrounding the agent [13], and agent moves closer to the wall but keeps away from the wall [45]. These two factors result in the highest density value $2.8 \mathrm{p} / \mathrm{m} 2$. As for the specific flow, the values are obtained with different mechanisms of measurement, but both the model values and calculated values perform at the same trend and agree with previous results.
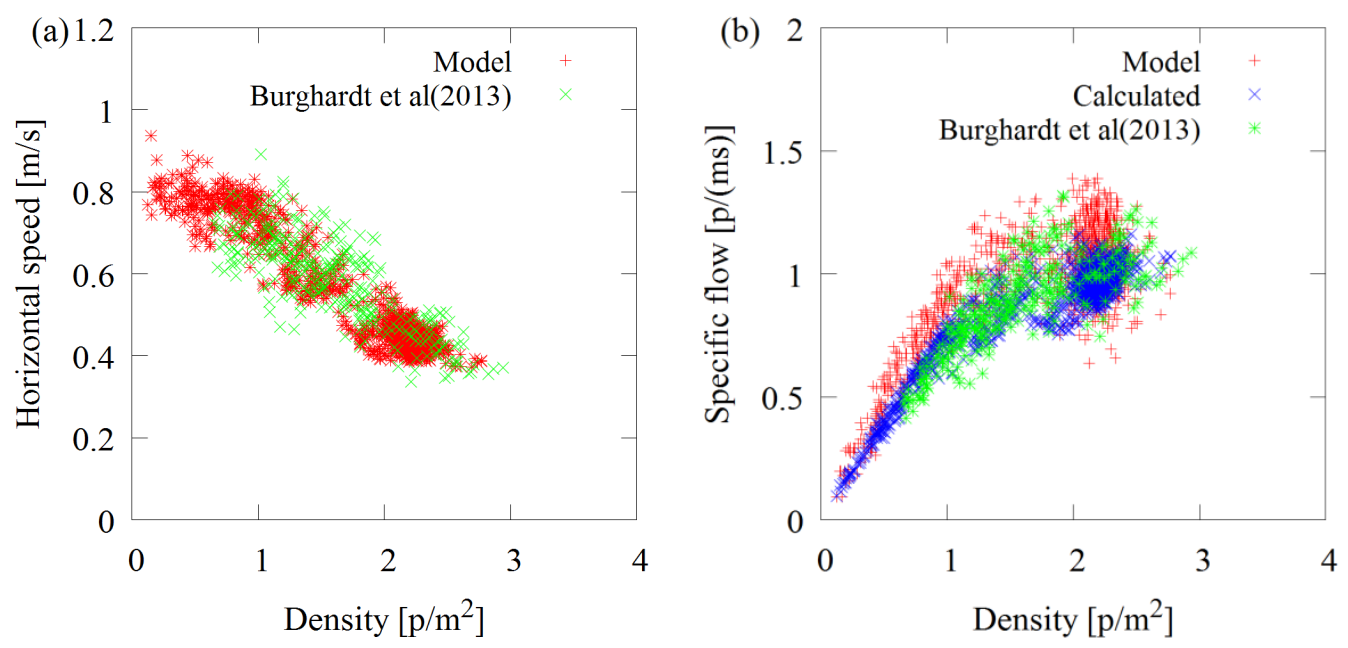

Fig. 11 The measurements of this model compared with value in Burghardt et al.[29]
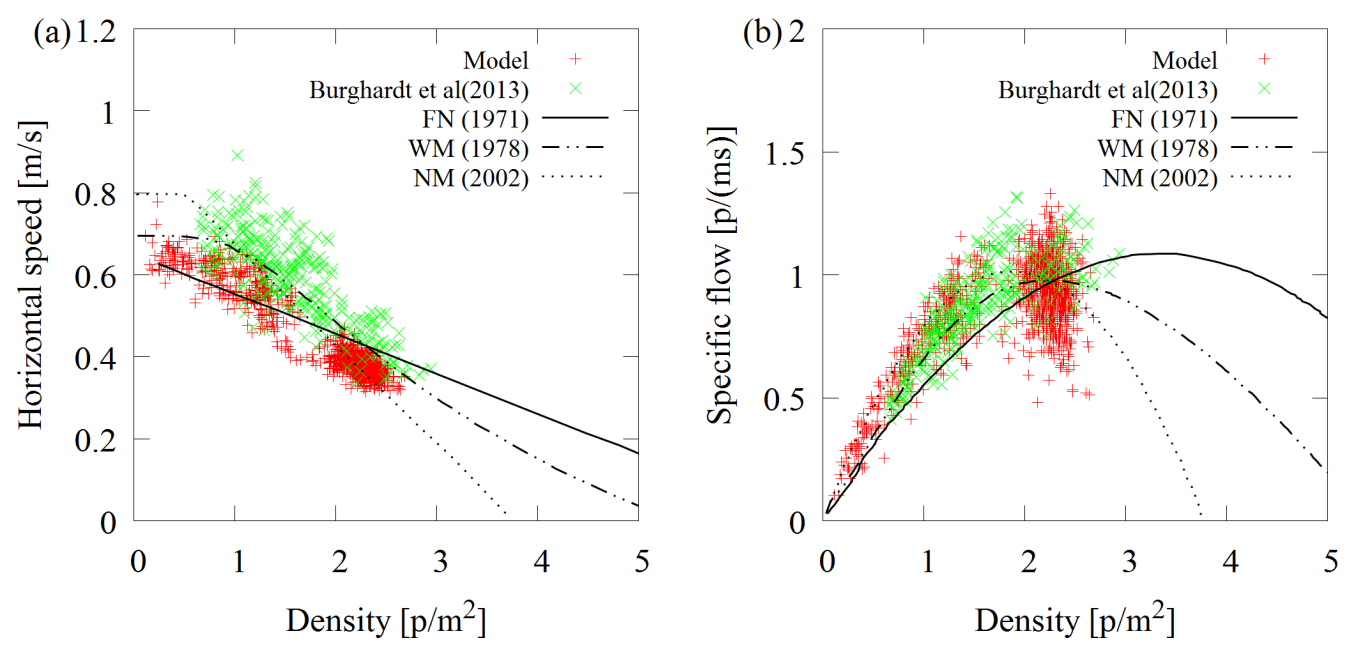

Fig. 12 Human performance with the approach of Lam and Cheung [46] value as input value

To further evaluate the validation of the model in respect of fundamental human performance, the values of Lam and Cheung [46] are considered as input variables. In paper of Lam and Cheung [46], people walked down stairs with horizontal velocity $0.601 \mathrm{~m} / \mathrm{s}$ in a stairwell where riser height $(\mathrm{mm})$, tread depth $(\mathrm{mm})$ and gradient (degree) are 150, 305 and 19.0, separately. From Fig. 12, although model results are a bit smaller than [29] in respect of fundamental diagram, the model results are in line with previous results. As for the specific flow, values from the model calculation are in accord with 
previous results when density is smaller than $3.0 \mathrm{p} / \mathrm{m}^{2}$; however, this kind of result cannot be obtained when density is larger than $3.0 \mathrm{p} / \mathrm{m}^{2}$. So in the near future, the model should be improved to make it applicable in high density situations.

\subsection{Validation based on lane forming behavior}

Lane formation is a self-organization behavior when occupants cluster together. Some researchers [47-49] found the phenomenon of lane formation. And Hoogendoorn and Daamen [50] thought lane forming was an important phenomenon in pedestrian dynamic movement when pedestrians were passing through a bottleneck. And a stair was considered as a kind of bottleneck, and lanes would be found when pedestrians passed through a staircase in [15]. In our model simulation, agents formed lanes by selforganization when they walked down stairs as shown in Fig. 13, which agrees with previous results [51]. At the green area in Fig. 13, pedestrians have preference to the inner side of the mid-landing due to the fact that generally pedestrians prefer to use the trajectory associated to the shortest distance $[17,52]$. And the arrows represent the direction of the pedestrians, which could form a bottleneck at this green area. For the purpose of analyzing the lane formation, the trajectories mappings are obtained by calculating the number on each grid when pedestrian pass through the stairs shown in Fig. 13 (b) and (c).

(a)

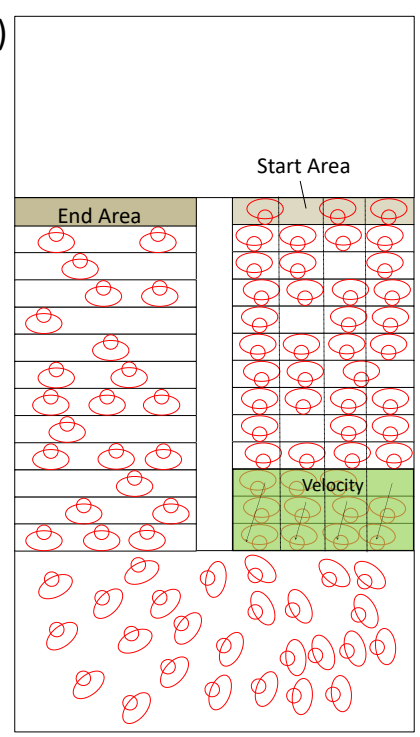

Frequency Distribution

(b)

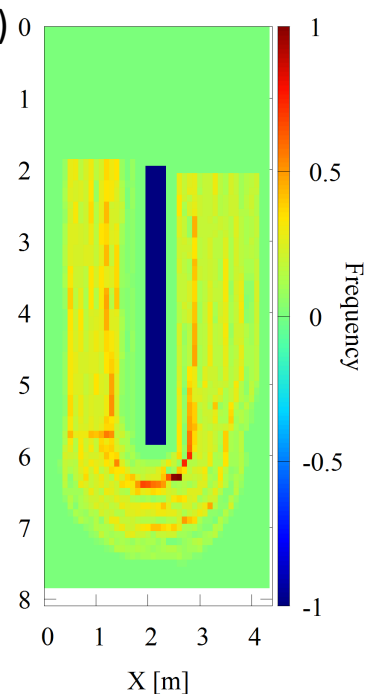

Frequency Distribution

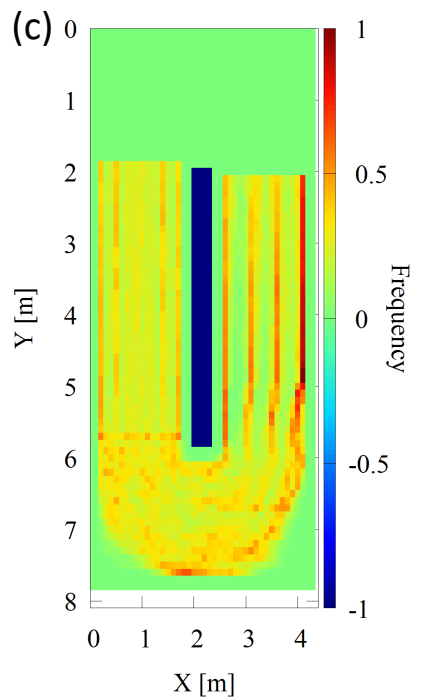

Fig. 13 A snapshot of lane formation in simulation(a) and trajectory mapping(b):lower density; (c): higher density)

It could be found that pedestrians are more willing to walk close to the inner side of the stairs [53] when they are reaching the mid-landing area. And at the lower density, lane formation phenomenon is not obvious while there are four lanes at the higher densities (probably due to the available space and typical spatial occupation of pedestrians). Furthermore, lane formation before mid-landing area is more obvious than 
that at other area due to shortest path. What is more, it seems that although agents have a preference for the inner side of the stairs, more pedestrians passed by the outside stairs from trajectory mapping at the higher density of Fig. 13. In order to quantify descriptions of the behavior, the frequency distributions at different densities are obtained and set up ranging from 0 to 1 as shown in Fig. 14: people are not present at the range [2.4:2.6] and [4.2:4.4] for two reasons: (1) the radius of agent is $0.2 \mathrm{~m}$ and the occupant are not allowed to compress in this model; (2) the locations of occupants are initiated within the range from $2.4 \mathrm{~m}$ to $4.2 \mathrm{~m}$. At lower density, most people prefer using the right side to pass through the end of stairs because the stairs presented in this model descend and rotate in a clockwise direction. Closer to the inner side agents are, density is higher. Although every agent has the preference to choose the inner side of the stair at lower density, the space is not sufficient to accommodate all the pedestrians, therefore some pedestrians are bound to use the outer lane to move to the destination as soon as possible. This phenomenon is even more visible at higher density situations, in which the distribution of pedestrian is not determined by shortest path and preference.

Considering the number of detected lanes, the stair can be divided into 4 parts to further investigate the pedestrian distribution: Fig. 15 shows the frequency distribution with four parts at the end of stairs. It is easily noted that at lower density most pedestrians use the inner side to evacuate, while most people reach at the mid-landing by the outside stairs at higher density, which is induced that outside of the stairs is necessary overall and even (counterintuitively) desirable from an individual perspective for evacuation at higher density. First of all, distance have potential effect on occupants, less distance contributes to less time taken on mid-landing, leading to more occupant passing by the stairs. Secondly, frequency distribution at the end of stairs is influenced by force. There is much space for occupant to move forward at lower density, so that less potential effect from other pedestrians is forced on the analyzing agent, while at higher density, the mid-landing is full of pedestrians and each occupant prefers to the shortest distance to evacuate, which results in that each agent close to the inner part of the stairs is greatly affected by the pedestrians surrounding the agent. In addition, compared to agents closer to the inner portion of stairs, occupants in the outer lanes have longer walking distance but they take advantage of less force, resulting in larger velocity at steady movement state. Our conjecture is that this does not represent an artifact of the modeling approach but that it rather accounts for the conflicts and slowdowns that occur in high density situations in reality. Nonetheless, additional empirical evidences should be gathered by experimental observations to back up this claim. 

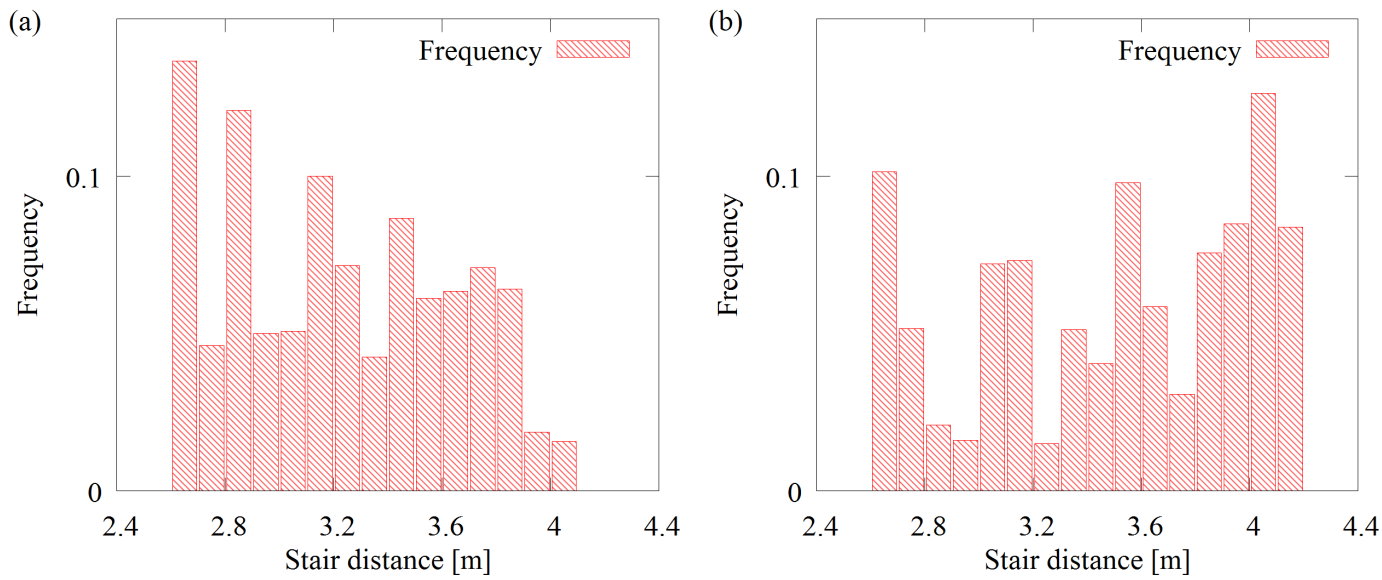

Fig. 14 Frequency distribution at the end of stairs: (a) lower density; (b) higher density
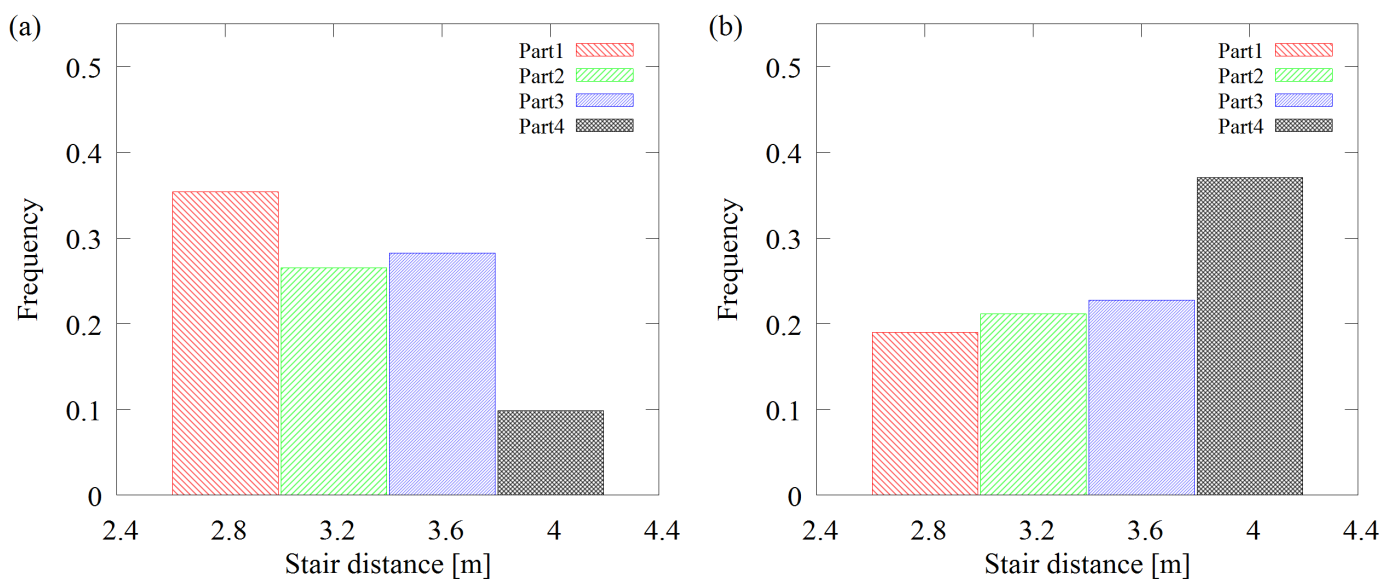

Fig. 15 Frequency distribution with four parts at the end of stairs: (a) lower density; (b) higher density

\subsection{The implication of the model}

In previous parts, the model is simulated to test the validation of the model in terms of fundamental diagram and lane formation. Then the model is implemented to analyze the effect of geometric structure on merging behaviors. It is demonstrated in previous studies that the merging area are in favor of floor stream when floor is connected adjacent to the incoming stairs $[12,17]$. Occupants from stairs are confronted with occupants only from Start Area a or Start Area b when they are placed in the following two scenarios, as shown in Fig. 15. 


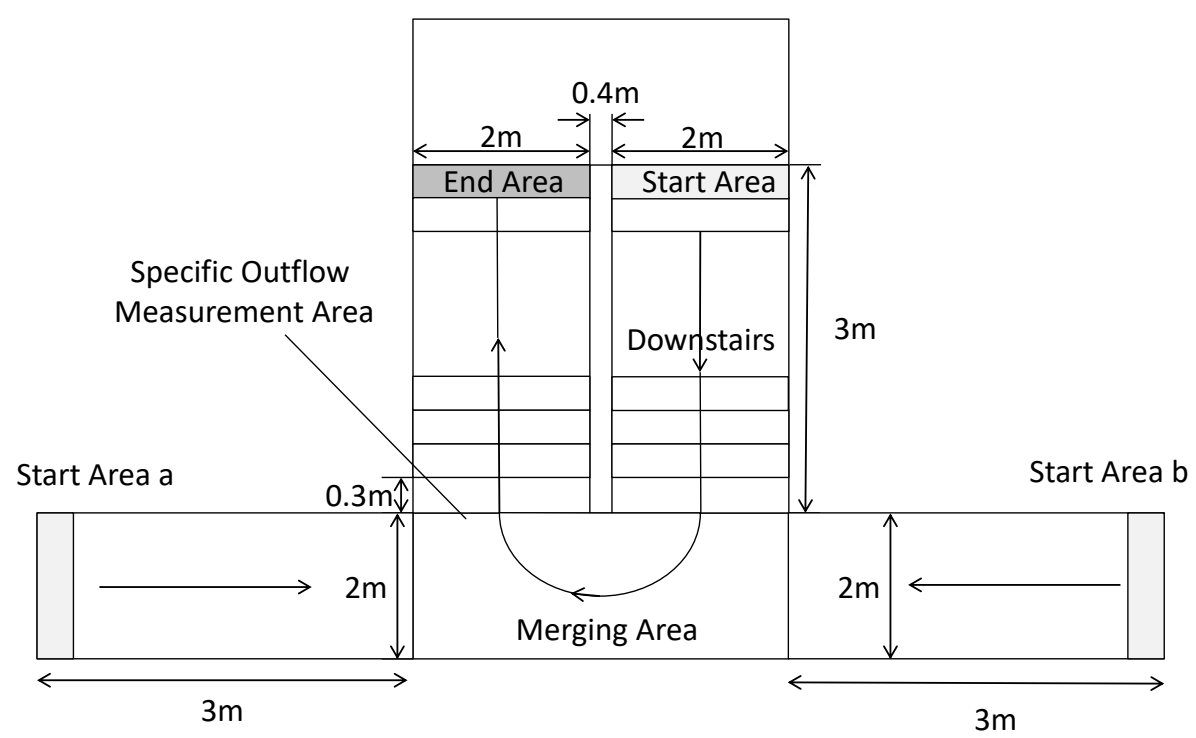

Fig. 16 Schematic of the pedestrian merging flow at the floor-stair interface. two geometric structures
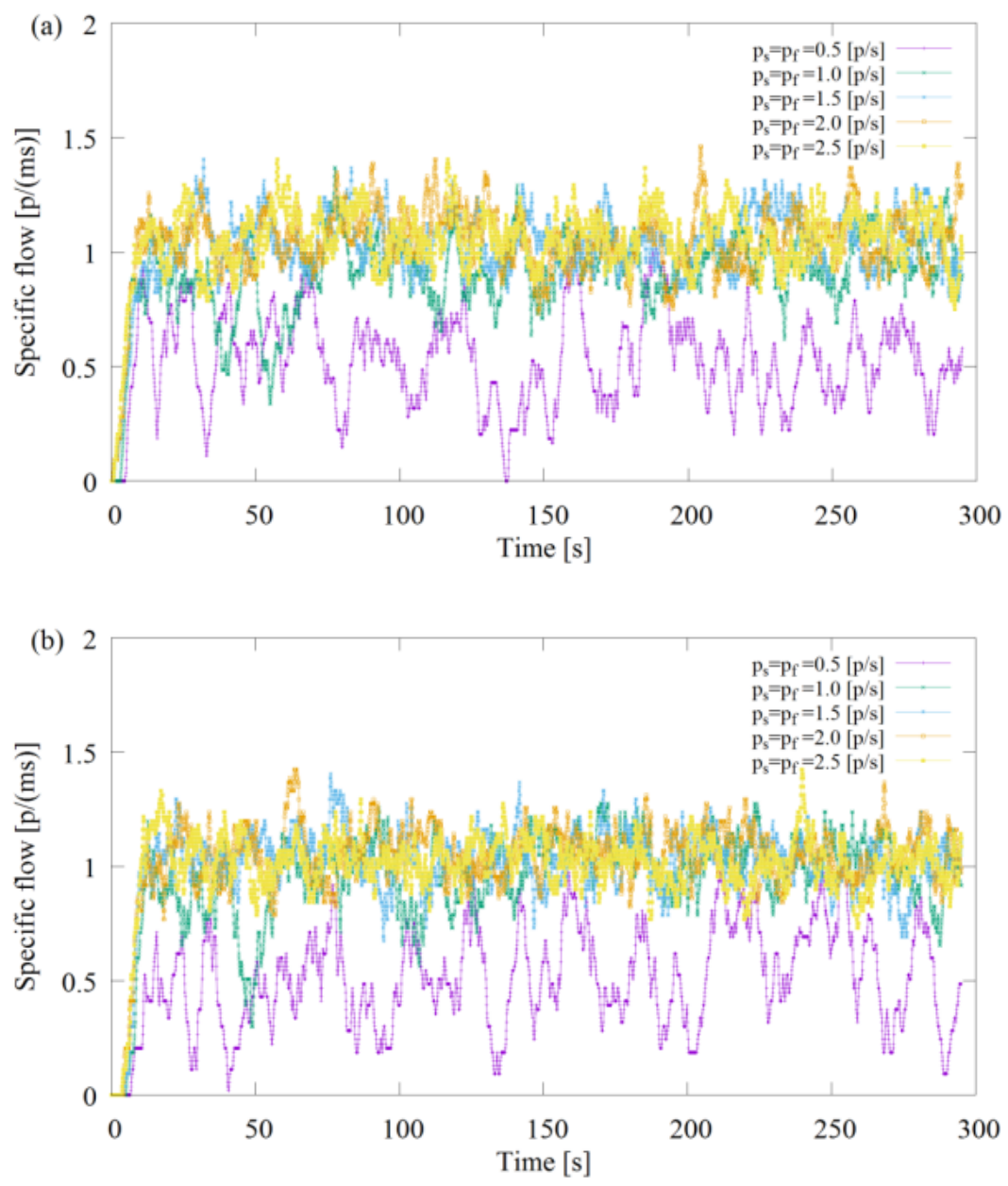

Fig. 17 Illumination of specific flow with time for the corridor (a) opposite and (b)adjacent to the incoming stairs 

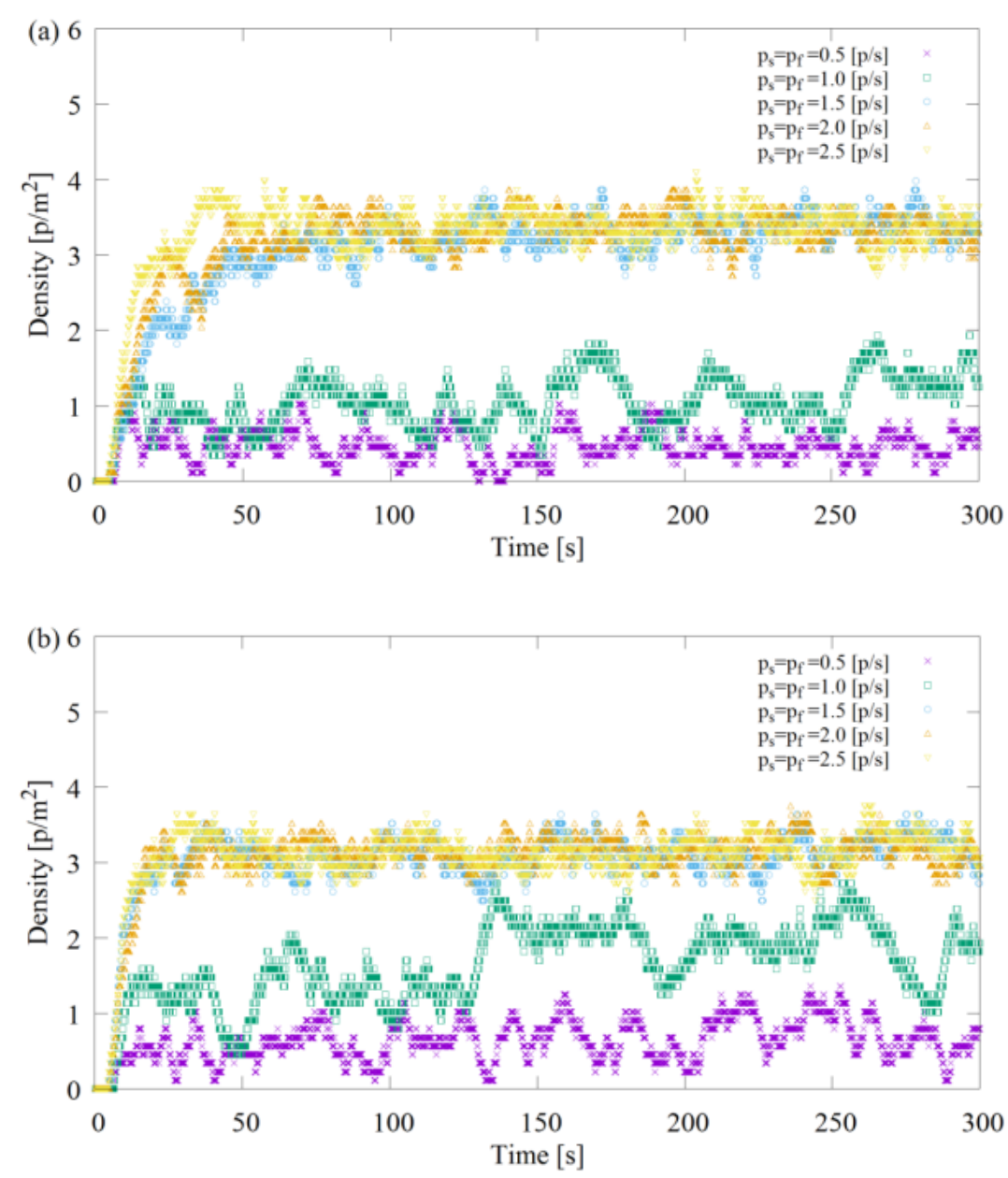

Fig. 18 changes of densities with time for the corridor (a) opposite and (b) adjacent to the incoming stairs

Pedestrian are generated with the same probability, that is occupants from stairs are equal to the number of agents from floors, $\mathrm{p}_{\mathrm{s}}=\mathrm{p}_{\mathrm{f}}$. For the purpose of investigating merging behaviors, it is necessary to obtain the results when system keeps a steady state. Fig. 17 shows specific outflow changes with time for the corridor (a) opposite and (b) adjacent to the incoming stairs. The result of specific flow ends with a value of $2.5[\mathrm{p} / \mathrm{s}]$ in order to show results clearly because the density at mid-landing keeps at a value of about $3 \mathrm{p} / \mathrm{m}^{2}$ with higher value than $2.5[\mathrm{p} / \mathrm{s}]$. Furthermore, the steady outflow value is about $1 \mathrm{p} /(\mathrm{ms})$, which agrees with the value of $0.99 \mathrm{p} /(\mathrm{ms})$ in [12].

Fig. 18 shows changes of densities with time for different merging geometry stairs structure. It is easily noted that when occupants are placed with a higher value of about $1.5[\mathrm{p} / \mathrm{s}]$, the densities do not change and the max density is about $3.0 \mathrm{p} / \mathrm{m}^{2}$. From Fig. 17 and Fig. 18, occupants move at a steady state when occupants are generated with a higher value of $1.5[\mathrm{p} / \mathrm{s}]$. Thus the outflow are compared when occupants generated is no less than 1.5 [p/s] in Fig. 19(a). And the specific outflow ratio (occupants from stairs: occupants from floor) are shown in Fig. 19(b). When the corridor is connected to the landing adjacent to the incoming stairs, the outflow of occupants from floor is larger 
than that from stairs, which demonstrates this structure is biased in favor of occupants from floors. However, when corridor is connected to the landing opposite to the incoming stairs, the outflow of occupants from floor is smaller than that from stairs, which indicates that this structure could retard the movement of occupants from floors. Merging performance of occupants is in accord with previous results. At lower densities, in particular for pedestrian generation smaller than $1.5 \mathrm{p} / \mathrm{s}$, there is no steady state, no congestion, and the outflow ratio shows that the stair capacity is sufficient to accommodate both flows.
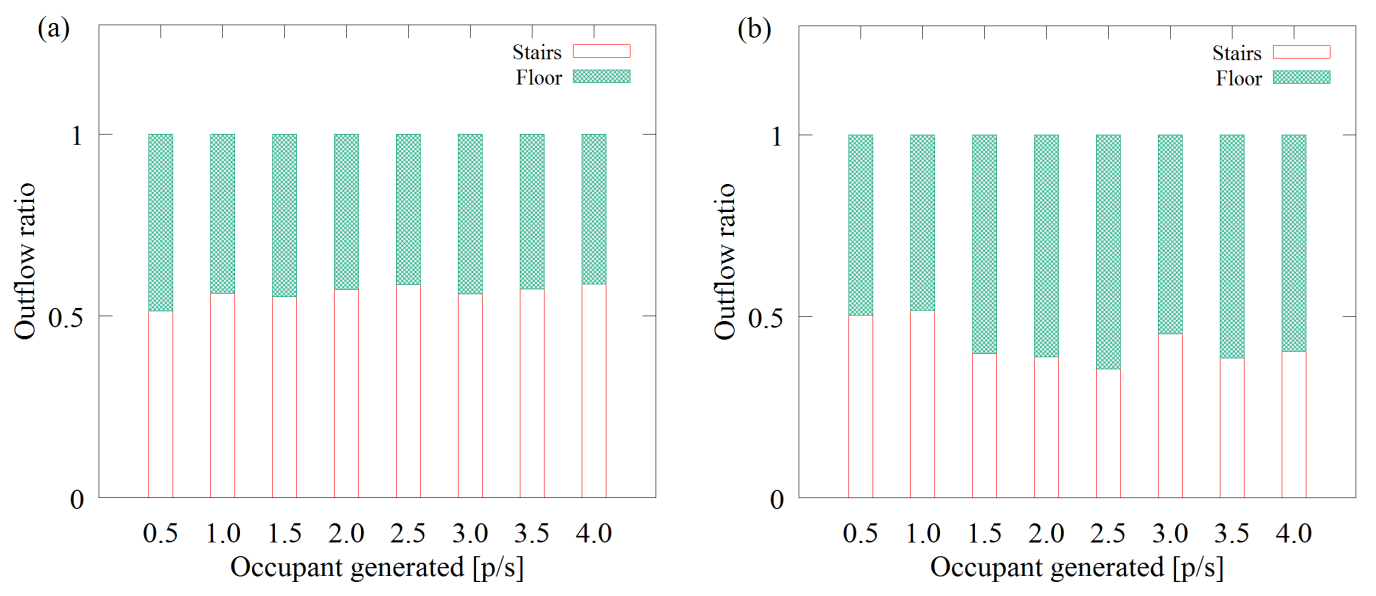

Fig. 19 Time evolution of the specific outflow ratio for the corridor (a) opposite and (b) adjacent to the incoming stairs

\section{Conclusion}

With more and more high-rise building springing into the cities, the movement on stairs is of great importance for the evacuation in high-rise buildings and the topic of human performance on stairs has attracted a lot of researcher to study. However, there is still a lack of knowledge about the research on stairs with mid-landing and merging behaviors in Optimal Steps Model. In this paper, Optimal Steps Model is modified by taking block-based floor field into consideration under open boundary conditions, in order to reproduce movement at mid-landing and study performance of occupants in stairwell.

Movements on stairs are simulated and studied with the help of extended model. The simulated results perform well and are in accord with previous research in respect of specific flow and fundamental diagram. Lane formations on stairs come into being before mid-landing area through simulations. And there are four lanes due to the limitation of stairs width and occupants radius. Furthermore, at higher density, the midlanding is full of pedestrians and each occupant prefers to the shortest distance to evacuate, which results in that each agent close to the inner stairs is greatly affected by other pedestrians. So that most people reach at the mid-landing by the outside stairs at higher density due to more space and less force from other agents, which is induced that outside of the stairs is good for evacuation. At last the model is implemented to study the effect of stairs geometry on merging behaviors. It is found that when the 
corridor is connected to the landing adjacent to the incoming stairs, the outflow of occupants from floor is larger than that from stairs, which demonstrates this structure is biased in favor of occupants from floors. However, when corridor is connected to the landing opposite to the incoming stairs, the outflow of occupants from floor is smaller than that from stairs, which indicates that this structure could retard the movement of occupants from floors. Merging performance of occupants is in accord with previous results. The work in this paper is intended to better understand movement during stair evacuations and develop a technical foundation for codes and standards requirements as well as egress modeling techniques.

\section{Acknowledgement}

This research was supported by Key Research and Development Program (2016YFC0802508), Program of Shanghai Science and Technology Committee (16DZ1200106), Specialized Research Fund for the Doctoral Program of Higher Education of China (20133402110009) and Fundamental Research Funds for the Central Universities (WK2320000035).

\section{Reference}

[1] E. Ronchi and D. Nilsson, "Fire evacuation in high-rise buildings: a review of human behaviour and modelling research," Fire science reviews, vol. 2, pp. 1-21, 2013.

[2] Code for Fire Protection Design of Tall Buildings [S], 2005.

[3] N. F. P. Association, NFPA 101 life safety code: National Fire Protection Association, 2011.

[4] T. Sano, M. Yajima, H. Kadokura, and A. Sekizawa, "Human behavior in a staircase during a total evacuation drill in a high-rise building," Fire and Materials, vol. 41, pp. 375-386, 2016.

[5] A. Leahy, "Observed Trends in Human Behavior Phenomena within High-Rise Stairwells," College Park, University of Maryland, 2011.

[6] J. J. Fruin, "Pedestrian Planning and Design. New York: Metropolitan Association of Urban Designers and Environmental Planners," ed, 1971.

[7] M. Kagawa, S. Kose, and Y. Morishita, "Movement Of People On Stairs During A Fire Evacuation Drill-japanese Experience In A Hgirise Office Building," Fire Safety Science, vol. 1, pp. 533-540, 1986.

[8] J.-h. Choi, H.-s. Hwang, and W.-h. Hong, "Predicting the probability of evacuation congestion occurrence relating to elapsed time and vertical section in a high-rise building," in Pedestrian and Evacuation Dynamics, ed: Springer, 2011, pp. 37-46.

[9] R. D. Peacock, B. L. Hoskins, and E. D. Kuligowski, "Overall and local movement speeds during fire drill evacuations in buildings up to 31 stories," Safety Science, vol. 50, pp. 1655-1664, Oct 2012.

[10] E. Kuligowski, R. Peacock, E. Wiess, and B. Hoskins, "Stair evacuation of older adults and people with mobility impairments," Fire Safety Journal, vol. 62, pp. 230-237, 2013.

[11] Z. G. Jiang and X. I. Liu, "Numerical Simulation Study on High-rise Student Apartment Fire Evacuation," Materials Science and Information Technology, Pts 1-8, vol. 433-440, pp. 3011- 
3016, 2012.

[12] E. R. Galea, G. Sharp, and P. J. Lawrence, "Investigating the Representation of Merging Behavior at the Floor-Stair Interface in Computer Simulations of Multi-Floor Building Evacuations," Journal of Fire Protection Engineering, vol. 18, pp. 291-316, Nov 2008.

[13] D. Helbing, I. Farkas, and T. Vicsek, "Simulating dynamical features of escape panic," Nature, vol. 407, pp. 487-490, Sep 282000.

[14] X. Song, Z. Zhang, G. Peng, and G. Shi, "Effect of authority figures for pedestrian evacuation at metro stations," Physica A: Statistical Mechanics and its Applications, vol. 465, pp. 599-612, 2017.

[15] Y. Qu, Z. Gao, Y. Xiao, and X. Li, "Modeling the pedestrian's movement and simulating evacuation dynamics on stairs," Safety Science, vol. 70, pp. 189-201, 2014.

[16] C. Wang and W. Weng, "Study on evacuation characteristics in an ultra high-rise building with social force model," in Intelligent Transportation Systems (ITSC), 2014 IEEE 17th International Conference on, 2014, pp. 566-571.

[17] F. Huo, W. Song, W. Lv, and K. Liew, "Analyzing pedestrian merging flow on a floor-stair interface using an extended lattice gas model," Simulation, vol. 90, pp. 501-510, 2014.

[18] D. Ning, Z. Hui, C. Tao, and P. B. Luh, "Stair evacuation simulation based on cellular automata considering evacuees' walk preferences," Chinese Physics B, vol. 24, Jun 2015.

[19] M. J. Seitz and G. Köster, "Natural discretization of pedestrian movement in continuous space," Physical Review E, vol. 86, p. 046108, 2012.

[20] I. von Sivers and G. Köster, "Dynamic stride length adaptation according to utility and personal space," Transportation Research Part B: Methodological, vol. 74, pp. 104-117, 2015.

[21] G. Köster, D. Lehmberg, and F. Dietrich, "Is Slowing Down Enough to Model Movement on Stairs?," in Traffic and Granular Flow'15, ed: Springer, 2016, pp. 35-42.

[22] X. Song, L. Ma, Y. Ma, C. Yang, and H. Ji, "Selfishness-and Selflessness-based models of pedestrian room evacuation," Physica A: Statistical Mechanics and its Applications, vol. 447, pp. 455-466, 2016.

[23] X. A. Xu and W. G. Song, "Staircase evacuation modeling and its comparison with an egress drill," Building and Environment, vol. 44, pp. 1039-1046, May 2009.

[24] "GB10000-88 China National Standard Human Dimensions of Chinese Adults."

[25] Z. M. Fang, W. G. Song, X. Liu, W. Lv, J. Ma, and X. Xiao, "A continuous distance model (CDM) for the single-file pedestrian movement considering step frequency and length," Physica aStatistical Mechanics and Its Applications, vol. 391, pp. 307-316, Jan 12012.

[26] T. Fujiyama and N. Tyler, "Predicting the walking speed of pedestrians on stairs," Transportation Planning and Technology, vol. 33, pp. 177-202, 2010.

[27] L. Yang, P. Rao, K. Zhu, S. Liu, and X. Zhan, "Observation study of pedestrian flow on staircases with different dimensions under normal and emergency conditions," Safety Science, vol. 50, pp. 1173-1179, 2012.

[28] J. Chen, J. Ma, and S. Lo, "Event-driven modeling of elevator assisted evacuation in ultra highrise buildings," Simulation Modelling Practice and Theory, vol. 74, pp. 99-116, 2017.

[29] S. Burghardt, A. Seyfried, and W. Klingsch, "Performance of stairs-fundamental diagram and topographical measurements," Transportation Research Part C: Emerging Technologies, vol. 37, pp. 268-278, 2013.

[30] F. Huo, W. Song, L. Chen, C. Liu, and K. M. Liew, "Experimental study on characteristics of 
pedestrian evacuation on stairs in a high-rise building," Safety Science, vol. 86, pp. 165-173, 2016.

[31] T. Fujiyama and N. Tyler, "An explicit study on walking speeds of pedestrians on stairs," pp. 643652, 2004.

[32] T. Fujiyama and N. Tyler, "Free walking speeds on stairs: Effects of stair gradients and obesity of pedestrians," in Pedestrian and Evacuation Dynamics, ed: Springer, 2011, pp. 95-106.

[33] L. Wang, M. Liu, and B. Meng, "Incorporating topography in a cellular automata model to simulate residents evacuation in a mountain area in China," Physica A: Statistical Mechanics and its Applications, vol. 392, pp. 520-528, 2013.

[34] J. L. Pauls, "Suggestions on evacuation models and research questions," in Proceedings of the 3rd International Symposium on Human Behavior in Fires, 2004, pp. 23-33.

[35] J. A. Sethian, "Level set methods and fast marching methods," Journal of Computing and Information Technology, vol. 11, pp. 1-2, 2003.

[36] J. A. Sethian, "A fast marching level set method for monotonically advancing fronts," Proceedings of the National Academy of Sciences, vol. 93, pp. 1591-1595, 1996.

[37] S. Li, X. Li, Y. Qu, and B. Jia, "Block-based floor field model for pedestrian's walking through corner," Physica A: Statistical Mechanics and its Applications, vol. 432, pp. 337-353, 2015.

[38] J. A. Templer, "Stair shape and human movement," Columbia University New York, 1974.

[39] H. Hyun-seung, C. Jun-ho, and H. Won-hwa, "Calculating and verifying the staircase-length for evacuation analysis," in Pedestrian and Evacuation Dynamics, ed: Springer, 2011, pp. 601-611.

[40] M. J. Seitz and G. Köster, "How update schemes influence crowd simulations," Journal of Statistical Mechanics: Theory and Experiment, vol. 2014, p. P07002, 2014.

[41] F. Huo, W. Song, L. Chen, C. Liu, and K. Liew, "Experimental study on characteristics of pedestrian evacuation on stairs in a high-rise building," Safety Science, vol. 86, pp. 165-173, 2016.

[42] J. H. Choi, E. R. Galea, and W. H. Hong, "Individual Stair Ascent and Descent Walk Speeds Measured in a Korean High-Rise Building," Fire Technology, vol. 50, pp. 267-295, Mar 2014.

[43] H. Nelson and F. Mowrer, "Emergency Movement, The SFPE Handbook of Fire Protection Engineering, ed," DiNenno P., Walton DW National Fire Protection Association, 2002.

[44] U. Weidmann, "Transporttechnik der Fussgänger. Institut für Verkehrsplanung, Transporttechnik, Strassen-und Eisenbahnbau," Schriftenreihe des IVT, 1993.

[45] J. Ma, W. G. Song, Z. M. Fang, S. M. Lo, and G. X. Liao, "Experimental study on microscopic moving characteristics of pedestrians in built corridor based on digital image processing," Building and Environment, vol. 45, pp. 2160-2169, Oct 2010.

[46] W. H. Lam and C.-y. Cheung, "Pedestrian speed/flow relationships for walking facilities in Hong Kong," Journal of transportation engineering, vol. 126, pp. 343-349, 2000.

[47] J. Zhang, W. Klingsch, A. Schadschneider, and A. Seyfried, "Ordering in bidirectional pedestrian flows and its influence on the fundamental diagram," Journal of Statistical Mechanics: Theory and Experiment, vol. 2012, p. P02002, 2012.

[48] S. Nowak and A. Schadschneider, "Quantitative analysis of pedestrian counterflow in a cellular automaton model," Physical Review E, vol. 85, p. 066128, 2012.

[49] C. Feliciani and K. Nishinari, "Empirical analysis of the lane formation process in bidirectional pedestrian flow," Physical Review E, vol. 94, p. 032304, 2016.

[50] S. Hoogendoorn and W. Daamen, "Self-organization in pedestrian flow," in Traffic and Granular 
Flow'03, ed: Springer, 2005, pp. 373-382.

[51] W. Lei, A. Li, R. Gao, and X. Wang, "Influences of exit and stair conditions on human evacuation in a dormitory," Physica A: Statistical Mechanics and its Applications, vol. 391, pp. 6279-6286, 2012.

[52] Y. Zeng, W. Song, S. Jin, R. Ye, and X. Liu, "Experimental study on walking preference during high-rise stair evacuation under different ground illuminations," Physica A: Statistical Mechanics and its Applications, vol. 479, pp. 26-37, 2017.

[53] J. D. Averill and W. Song, Accounting for emergency response in building evacuation: modeling differential egress capacity solutions: US Department of Commerce, Technology Administration, National Institute of Standards and Technology, Building and Fire Research Laboratory, 2007. 